\title{
Integration of Shape and a Multihypotheses Fisher Color Model for Figure-Ground Segmentation in Non-Stationary Environments
}

\author{
Francesc Moreno-Noguer Alberto Sanfeliu \\ Institut de Robòtica i Informàtica Industrial, UPC-CSIC \\ Llorens Artigas 4-6, 08028, Barcelona, Spain \\ fmoreno,asanfeliu@iri.upc.es
}

\begin{abstract}
In this paper a new technique to perform figure-ground segmentation in image sequences of scenarios with varying illumination conditions is proposed. The set of color points of both the target and background are modelled with Mixture of Gaussians (MoG), which optimum number is automatically initialized. Based on the 'Linear Discriminant Analysis' (LDA) a new colorspace that maximizes the foreground/background class separability is presented. Moreover, there is no need to assume gradual change of the viewing conditions over time, because the method works with multiple hypotheses about the next state of the color distribution (some considering small changes and other more abrupt variations). The hypothesis that generates the best object segmentation and the shape information in the previous iteration are fused to accurately detect the object boundary, in a stage denominated 'sample concentration', introduced as a final step to the classical CONDENSATION algorithm.
\end{abstract}

\section{Introduction}

Color is a visual cue that is commonly used in computer vision applications such as object detection and tracking tasks. In environments with controlled lighting conditions and uncluttered background, color can be considered a robust and invariant cue, but when dealing with real scenes with changing illumination and confusing backgrounds, the apparent color of the objects varies considerably over time. In the literature, the techniques that cope with change in color appearance can be divided in two groups. On the one side, there are the approaches searching for color constancy (e.g. [2]); but in practice, these methods work on artificial and highly constrained environments. On the other hand, there are the techniques that generate a stochastic model

0 This work was supported by CICYT projects DPI2001-2223 and DPI2000-1352-C02-01, and by a fellowship from the Spanish Ministry of Science and Technology. of the color distribution, and adapt it over time [7], [8]. The drawback in all these approaches is that they assume a smooth and slow color variation that can be predicted by a dynamic model based in only one hypothesis (usually by a weighting function). However, this assumption does not suffice to cope with general scenes, where the dynamics of the color distribution might follow an unknown an unpredictable path. In a previous work [6], we have suggested the use of a multihypotheses framework to track color objects in such situations, where the color could be approximated by an unimodal distribution. In the present work, we deal with multicolored objects.

An overview of the system is given in Section 2. In Section 3 we describe the Fisher colorspace. Sections 4 and 5 present the color and dynamical models. The complete tracking algorithm is depicted in Section 6. Results and conclusions are presented in Sections 7 and 8, respectively.

\section{System Overview}

In order to cope with unconstrained environments, we propose a system with the following main features, that represent contributions with respect to previous works:

- Fisher color model: Instead of using the classical $R G B, r g b^{1}, X Y Z$ or $H S V$ colorspaces, we propose the use of a colorspace efficient for the discrimination between foreground and background classes, based on the 2D projection of the $R, G$ and $B$ components on the plane obtained from a nonparametric $L D A$ [4].

- Multihypotheses framework: The use of a particle filter formulation to predict the color distribution in following iterations, offers robustness to abrupt and unexpected changes in the color appearance.

- Integration of color and shape: The fusion of both vision modules is done in a final stage introduced to the classical CONDENSATION algorithm, and makes our method suitable to work in cluttered scenes.

1 Lowercase letters represent normalized components 


\section{Fisher Colorspace}

The selection of the colorspace is an important initial issue for any color-based figure-ground segmentation system. The typical selection criterion is based on the invariance of the color representation to illumination changes, and according to this idea, color is usually represented by two components of the $r g b, H S V$ or $x y z$ colorspaces. However, these representations are not robust enough to cope with abrupt illumination changes. In this paper we propose a different criterion and select a $2 \mathrm{D}$ colorspace that maximizes the separability of the object and background classes.

Let $\mathbf{x}$ be a $3 \mathrm{D}$ vector with the color value of image pixels in $R G B$ space, which must be classified as foreground $\left(\omega_{f}\right)$ or background $\left(\omega_{b}\right)$. When we are dealing with multicolored objects, the parameterization of color distributions in $3 \mathrm{D}$ colorspace becomes very complex. To simplify, we reduce the dimensionality to $2 \mathrm{D}$ by projecting the data on a plane $\boldsymbol{\Phi}=\left[\phi_{1}, \phi_{2}\right] \in \mathcal{M}_{3 \times 2}$, that is, $\mathbf{y}=\mathbf{\Phi} \mathbf{x}$, where $\mathbf{y}$ are the $2 \mathrm{D}$ linearly transformed coordinates used for classification. The most popular way to find the best linear features is the parametric version of the $L D A$ method, where training data is used to construct the within-class $S_{w}$ and betweenclass $S_{b}$ scatter matrices, in the $c$-class problem defined as,

$$
\begin{aligned}
S_{w} & =\sum_{i=1}^{c} P\left(\omega_{i}\right) E\left[\left(\left.\mathbf{x}\right|_{\omega_{i}}-\mu_{i}\right)\left(\left.\mathbf{x}\right|_{\omega_{i}}-\mu_{i}\right)^{T}\right]=\sum_{i=1}^{c} P\left(w_{i}\right) S_{i} \\
S_{b} & =\sum_{i=1}^{c} P\left(\omega_{i}\right) E\left[\left(\mathbf{x}-\mu_{o}\right)\left(\mathbf{x}-\mu_{o}\right)^{T}\right]
\end{aligned}
$$

where $P\left(\omega_{i}\right)$ is the prior of the $\mathrm{i}^{t h}$ class, $\mu_{i}$ and $S_{i}$ are its expected vector and covariance matrix, $\mu_{o}$ is the overall mean and $\left.\mathbf{x}\right|_{\omega_{i}}$ indicates that sample $\mathbf{x}$ belongs to $\omega_{i}$.

A typical criterion of class separability is formulated by maximizing $J=\operatorname{trace}\left(\left(\boldsymbol{\Phi}^{T} S_{w} \boldsymbol{\Phi}\right)^{-1}\left(\boldsymbol{\Phi}^{T} S_{b} \boldsymbol{\Phi}\right)\right)$, and seeks for the separation of the class means in the transformed $Y$-space (high $S_{b}$ ), while the classes remain compact (small $S_{w}$ ). The classical $L D A$ maximizes $J$ by constructing the columns of $\boldsymbol{\Phi}$ with the eigenvectors of $S_{w}^{-1} S_{b}$ having the highest eigenvalues.

As the maximum rank of $S_{b}$ is $c-1$, this will be the maximum dimension of the projected $Y$-space. This limitation can be solved by the nonparametric LDA [4], that computes $S_{b}$ using local information and the $k$ Nearest Neighbors $(\mathrm{KNN})$ rule. In the 2-class problem that we have in hands, this matrix (denoted $\Sigma_{b}$ ) is defined as,

$$
\begin{aligned}
\Sigma_{b} & =\frac{1}{N} \sum_{i=1}^{N_{f}} w_{i}\left(\left.\mathbf{x}_{i}\right|_{\omega_{f}}-M_{b}^{k}\left(\left.\mathbf{x}_{i}\right|_{\omega_{f}}\right)\right)\left(\left.\mathbf{x}_{i}\right|_{\omega_{f}}-M_{b}^{k}\left(\left.\mathbf{x}_{i}\right|_{\omega_{f}}\right)\right)^{T} \\
& +\frac{1}{N} \sum_{i=1}^{N_{b}} w_{i}\left(\left.\mathbf{x}_{i}\right|_{\omega_{b}}-M_{f}^{k}\left(\left.\mathbf{x}_{i}\right|_{\omega_{b}}\right)\right)\left(\left.\mathbf{x}_{i}\right|_{\omega_{b}}-M_{f}^{k}\left(\left.\mathbf{x}_{i}\right|_{\omega_{b}}\right)\right)^{T}
\end{aligned}
$$

where $N_{f}$ and $N_{b}$ are the number of samples of $\omega_{f}$ and $\omega_{b}$, $N=N_{f}+N_{b}, M_{j}^{k}\left(\mathbf{x}_{i}\right)$ is the mean of the $k N N$ in $\omega_{j}$ to a

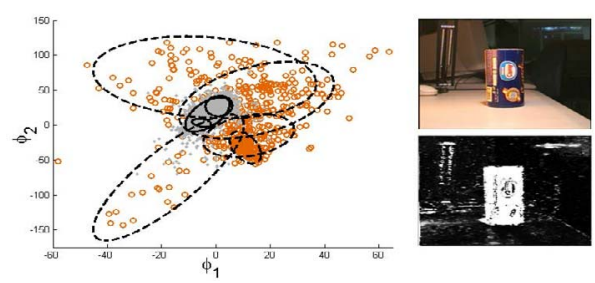

Figure 1. MoG of $\omega_{f}$ (the can) and $\omega_{b}$. Left image: empty dots and dashed lines are $\omega_{f}$ data (projected on $\Phi$ ) and Gaussian components, respectively. Filled dots and continuous lines are $\omega_{b}$ data and Gaussians. Lower right image: $p\left(\omega_{f} \mid \mathbf{y}\right)$, where brighter points correspond to more likely pixels.

point $\mathbf{x}_{i}$, and $w_{i}$ is a weighting function for deemphasizing samples far from the classification boundary (see [4]).

Given two sets $\left\{\mathbf{x}_{1}, \cdots, \mathbf{x}_{N_{f}} \mid \omega_{f}\right\},\left\{\mathbf{x}_{1}, \cdots, \mathbf{x}_{N_{b}} \mid \omega_{b}\right\}$ of $R G B$ pixel values used as training data, the optimum linear mapping is obtained with the following steps:

- Calculate $S_{w}$ with eq. 1 and whiten the data with respect to it. That is, transform $\mathbf{x}$ to $\mathbf{z}=\Lambda^{-1 / 2} \Omega^{T} \mathbf{x}$, where $\Lambda$ and $\Omega$ are the eigenvalue and eigenvector matrices of $S_{w}$.

- Select $k$ and (in the $Z$-space) compute $\Sigma_{b}$ using eq. 2 .

- Select the two eigenvectors $\Psi_{1}, \Psi_{2}$ of $\Sigma_{b}$ with the two largest eigenvalues.

- The optimum linear mapping from the original $R G B$ space to the discriminant subspace (we call it Fisher colorspace) is given by $\mathbf{y}=\Psi^{T} \Lambda^{-1 / 2} \Omega^{T} \mathbf{x}$.

In the Results Section it will be shown that with this colorspace we obtain better rates in pixel classification.

\section{Color Model and Parameterization}

The color distributions of fore and background transformed to the Fisher colorspace, are represented by a Gaussian mixture model. The conditional probability for a pixel $\mathbf{y}$ belonging to $\omega_{f}$ is expressed as a sum of $M_{f}$ Gaussian components as $p\left(\mathbf{y} \mid \omega_{f}\right)=\sum_{j=1}^{M_{f}} p(\mathbf{y} \mid j) P(j)$. Similarly, the background color distribution will be represented by a mixture of $M_{b}$ Gaussians (Fig. 1).

Next, the a posteriori probability that a pixel y belongs to the $\omega_{f}$ class is computed using the Bayes rule:

$$
p\left(\omega_{f} \mid \mathbf{y}\right)=\frac{p\left(\mathbf{y} \mid \omega_{f}\right) P\left(\omega_{f}\right)}{p\left(\mathbf{y} \mid \omega_{f}\right) P\left(\omega_{f}\right)+p\left(\mathbf{y} \mid \omega_{b}\right) P\left(\omega_{b}\right)}
$$

One of the problems when using $M o G$ is the selection of the number of components that better adjust the data. We initialize this, with the modified $E M$ algorithm proposed in [3], that is based on a Minimum Message Length criteria and iteratively fits and annihilates and initially large number of components (introduced by the user). Once we have 


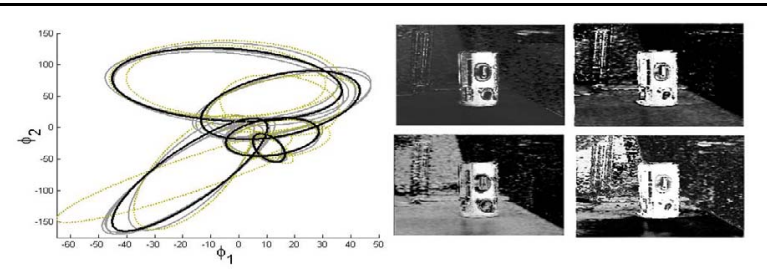

Figure 2. Left: Various predictions for the foreground MoG of Fig. 1. Continuous wider lines: MoG from previous iteration. Continuous narrower lines: MoG predicted with a model accounting for smooth changes. Dashed lines: predictions done with a model accounting for abrupt changes. Right images: $p\left(\omega_{f} \mid \mathbf{y}\right)$ for several predictions.

learnt the initial configurations for the $M o G$ of $\omega_{f}$ and $\omega_{b}$, they are parameterized by:

$$
\mathcal{X}_{\varepsilon}=\left[\mathbf{p}_{\varepsilon}, \mu_{\varepsilon}, \lambda_{\varepsilon}, \theta_{\varepsilon}\right]
$$

where $\varepsilon=\{f, b\}, \mathbf{p}_{\varepsilon}$ contains the priors for each Gaussian component, $\mu_{\varepsilon}$ the centroids, $\lambda_{\varepsilon}$ the eigenvalues of the principal directions and $\theta_{\varepsilon}$ the angles between the principal axis of each component with the horizontal.

\section{Learning the Dynamical Models}

In the tracking algorithm, we need to propagate the state vector (eq. 4) in order to generate multiple hypotheses about the future configurations of the $M o G$. These predictions are done by using the following dynamic motion model,

$$
\mathcal{X}_{\varepsilon, t}=A_{0} \mathcal{X}_{\varepsilon, t-2}+A_{1} \mathcal{X}_{\varepsilon, t-1}+B_{0} \mathbf{w}_{t}
$$

where the matrices $A_{0}, A_{1}$ represent the deterministic component of the model and $B_{0} \mathbf{w}_{t}$ is the stochastic part (learned a priori using the standard $M L E$ algorithm described in [1]). In practice, we define two kinds of dynamical models, one taking in account smooth changes and another that considers more abrupt variations (see Fig. 2).

\section{The Tracking Algorithm}

The basic steps of our tracking algorithm follow the procedure of the particle filters, but we introduce a modification to the classical CONDENSATION algorithm (similar to the ICONDENSATION technique [5]), and in order to 'direct' the search for the next iteration we add a final stage that concentrates the future hypotheses on those areas of the state-space containing more information about $p\left(\omega_{f} \mid \mathbf{y}\right)$. Moreover, in this final stage we fuse object color and shape information. Next, we present the steps of our algorithm:

- pdf of color distribution: At time $t$, a set of $N$ samples $\mathcal{S}_{t-1}^{(n)}(n=1, \ldots, N)$ with the same structure than $\mathcal{X}$ (eq. 4), parameterizing $N$ color distributions, are available from the previous iteration. Each

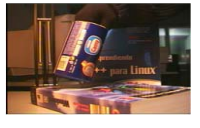

(a)

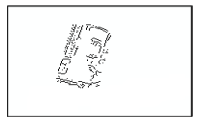

(d)

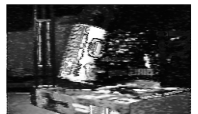

(b)

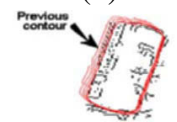

(e)

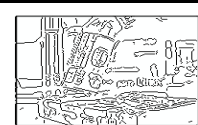

(c)

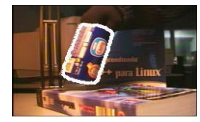

(f)
Figure 3. Steps to extract the exact position of the object fusing color segmentation and accurate adjustment by affine snakes (commented in the text).

sample has an associated weight $\pi_{t-1}^{(n)}$. The whole set represents an approximation to $p\left(\mathcal{X}_{t-1} \mid \mathcal{Z}_{t-1}\right)$ where $\mathcal{Z}_{t-1}=\left\{z_{0}, \ldots, z_{t-1}\right\}$ is the history of the measurements. The goal of the algorithm consists on construct a new sample set $\left\{\mathcal{S}_{t}^{(n)}, \pi_{t}^{(n)}\right\}$ to estimate $p\left(\mathcal{X}_{t} \mid \mathcal{Z}_{t}\right)$.

- Sampling from $p\left(\mathcal{X}_{t-1} \mid \mathcal{Z}_{t-1}\right)$ : A sampling with replacement is performed $N$ times on the set $\left\{\mathcal{S}_{t-1}^{(n)}\right\}$, where each element has probability $\pi_{t-1}^{(n)}$ of being chosen. This, will give us a set $\left\{\mathcal{S}_{t-1}^{\prime(n)}\right\}$.

- Probabilistic propagation of the samples: Each sample $\mathcal{S}_{t-1}^{(n)}$ is propagated to $\tilde{\mathcal{S}}_{t}^{(n)}$, using one of the learned dynamic models (eq. 5):

- Measure and Weight: Each element $\tilde{\mathcal{S}}_{t}^{(n)}$ has to be weighted according to some measured features. From the propagated samples $\tilde{\mathcal{S}}_{t}^{(n)}$ we construct the corresponding $M o G$, that are used to calculate $p\left(\omega_{f} \mid \mathbf{y}\right)$ for the whole image in Fisher colorspace, using eq. 3 . The weight assigned to each sample is $\pi_{t}^{(n)}=\frac{\sum_{\mathbf{y} \in W} p\left(\omega_{f} \mid \mathbf{y}\right)}{N_{w}}-\frac{\sum_{\mathbf{y} \notin W} p\left(\omega_{f} \mid \mathbf{y}\right)}{\overline{N_{w}}}$, where $W$ is the interest region around the previous object position, and $N_{w}, \overline{N_{w}}$ are the number of image pixels in and out of this interest region.

- Sample Concentration: In the last stage of our algorithm, we concentrate the samples around the local maxima, so that in the following iteration the hypotheses are formulated around these more likely regions of the state space. In our case, this is absolutely necessary because the state vector $\mathcal{X}$ has high dimensionality, and if we let the samples move freely, uniquely governed by the dynamic model, the number of hypotheses needed to find the samples representing a correct color configuration, is extremely high.

In this 'concentration' stage, firstly, the maximum from the set of weights $\left\{\pi_{t}^{(n)}\right\}$ is taken, and by morphologic operations on its $p\left(\omega_{f} \mid \mathbf{y}\right)$ map (Fig. 3b), a coarse approximation of the object shape is obtained. This, lets to eliminate noisy edges from the original 


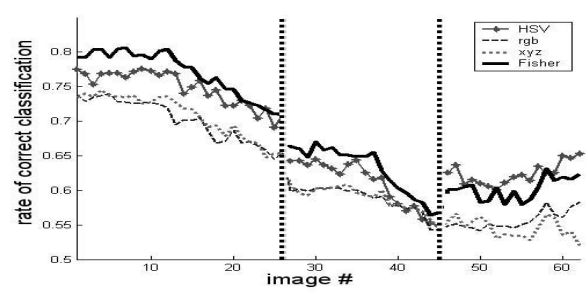

Figure 4. Classification results obtained with various colorspaces (3 different experiments).

image (Fig. 3c,d). Next, the contour of the object in the previous iteration, is used as initialization of an affine snake, that is adjusted (only by affine deformations) to the image of refined edges (Fig. 3e). The fusion of color and shape information increases the robustness of the system, because even when the color hypotheses give a highly rough estimation, they can be corrected using the contour information. Once the boundary of the object has been accurately detected (Fig. 3f), a $M o G$ is fitted to its color distribution (using the EM algorithm), giving a state vector $\mathcal{S}_{t}^{*}$. Samples $\left\{\tilde{\mathcal{S}}_{t}^{(n)}\right\}$ are 'concentrated' on this new distribution with the equation $S_{t}^{(n)}=(1-a) \widetilde{S}_{t}^{(n)}+a \mathcal{S}_{t}^{*}$, where the parameter $a$ governs the level of concentration.

\section{Results}

We start this Section by comparing the class discrimination power of the Fisher colorspace with other colorspaces (two components of the $r g b, H S V$ or $x y z$ ). To quantify the notion of class separability, a constant number of Gaussians are fitted to $\omega_{f}$ and $\omega_{b}$ distributions of hand segmented images, for each one of the colorspaces. Next, according to eq. 3 we segment the same images, assigning each pixel $\mathbf{y}$ to the class with maximal $p\left(\omega_{f} \mid \mathbf{y}\right)$. This result is compared with the hand segmented ones. In Fig. 4 we show the results for three different sequences, where the vertical axis represents the percentage of pixels well classified. The best results are obtained with the Fisher colorspace $(68.1 \%$ of correct classification), followed by the $H V$ components of $H S V(67.1 \%)$.

Next, it is shown the performance of the tracking system in two different situations. Fig. 5 represents three samples of the results obtained on a sequence with a gradual change of illumination and object position. In the second experiment (Fig. 6), there is an abrupt change of both illumination and object position (Fig. 6a,b are the two consecutive frames). At least one of the multiple hypotheses of color distributions performs a good a posteriori probability map (Fig. 6c) that is used to fit the contour (Fig. 6d,e).

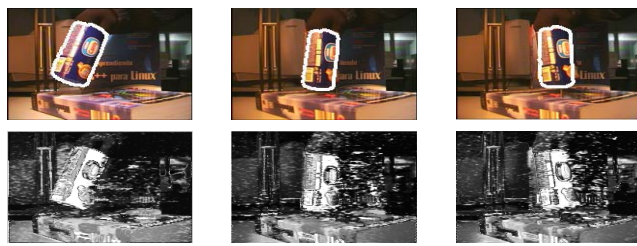

Figure 5. Fitted contour and $p\left(\omega_{f} \mid \mathbf{y}\right)$ map on a non-stationary sequence.

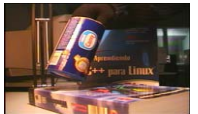

(a)

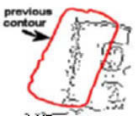

(d)

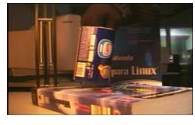

(b)

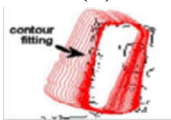

(e)

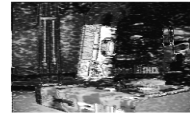

(c)

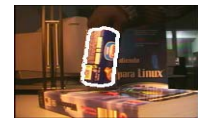

(f)
Figure 6. Results of an abrupt change of illuminant and object position (commented in text).

\section{Conclusions}

In this paper, it has been presented a new approach to figure-ground segmentation in non-stationary environments. We have introduced the concept of Fisher colorspace, that has better object/background discrimination performance than typical colorspaces, and the fusion of shape and color information in the probabilistic multiple hypotheses framework of the particle filters. This integration increases the robustness of the method, and is done in a last stage denominated 'sample concentration' that we have added to the CONDENSATION algorithm.

\section{References}

[1] A.Blake, M.Isard, Active contours. Springer, 1998.

[2] G.D.Finlayson, B.V.Funt, K.Barnard. Color Constancy under Varying Illumination. Proc. ICCV, pp.720-725, 1995.

[3] M.A.T.Figueiredo, A.K.Jain. Unsupervised Learning of Finite Mixture Models. PAMI, Vol.24, num.3, pp.381-396, 2002.

[4] K.Fukunaga. Introduction to Statistical Pattern Recognition (2nd. Edition) Academic Press, 1990.

[5] M.Isard, A.Blake Icondensaton: Unifying Low-Level and High-Level Tracking in a Stochastic Framework. Proc. ECCV, Vol.1, pp.893-908, 1998.

[6] F.Moreno-Noguer, J.Andrade-Cetto, A.Sanfeliu. Fusion of Color and Shape for Object Tracking under Varying Illumination. Proc.IBPRIA, LNCS 2652, Springer, pp.580-588, 2003.

[7] Y.Raja, S.McKenna, S.Gong. Colour Model Selection and Adaption in Dynamic Scenes. Proc. ECCV, Vol.1, pp.460 475, 2000.

[8] L.Sigal, S.Sclaroff, V.Athitsos. Estimation and Prediction of Evolving Color Distributions for Skin Segmentation under Varying Illumination. Proc. CVPR, Vol.2, pp.152-159, 2000. 